\title{
Cobertura Máxima de Redes de Sensores Inalámbricos para un Sistema de Gestión de Energía en Hogares Inteligentes
}

\section{Maximum Coverage of Wireless Sensor Networks for an Energy Management System in Smart Homes}

DOI: http://dx.doi.org/10.17981/ingecuc.12.2.2016.07

Artículo de Investigación Científica - Fecha de Recepción: 31 de Enero de 2016, Fecha de Aceptación: 28 de Junio de 2016

\author{
Paúl Alexander Andrade Montoya \\ Universidad Politécnica Salesiana Quito, Ecuador \\ pandradem@est.ups.edu.ec \\ José Luis Morejón Bastidas \\ Universidad Politécnica Salesiana Quito, Ecuador \\ jmorejonb@est.ups.edu.ec \\ Esteban Mauricio Inga Ortega \\ Universidad Politécnica Salesiana Quito, Ecuador \\ einga@ups.edu.ec
}

Cómo citar este artículo:

P. Andrade, J. Morejón y E. Inga. "Cobertura Máxima de Redes de Sensores Inalámbricos para un Sistema de Gestión de Energía en Hogares Inteligentes”, INGE CUC, vol. 12, No. 2, pp.68-78, 2016. DOI: http://dx.doi.org/10.17981/ingecuc.12.2.2016.07

\begin{abstract}
Resumen- Este artículo se desarrolla con base en redes de sensores sobre Smart Grids, en conjunto con una metodología de gestión apropiada que nos permita analizar y comprobar mediante una simulación el comportamiento de estas redes inalámbricas de una manera interna, dentro de una vivienda $u$ hogar inteligente, teniendo en cuenta varios parámetros o restricciones como son la distancia de cobertura, la capacidad y el porcentaje de cobertura. Para ello se describe la infraestructura de redes inteligentes y se plantea un modelo matemático para minimizar la distancia de los sensores a los puntos de acceso dentro del área considerada. Además está enfocado en el diseño de estas redes inteligentes, donde se realiza una optimización de los diferentes puntos factibles, con lo que se logra una máxima cobertura de los sensores para implementar un adecuado sistema de gestión de energía en hogares inteligentes, basado en un servicio fiable de la conectividad de la red.
\end{abstract}

Palabras clave-Redes de Sensores Inalámbricos, Hogares Inteligentes, Cobertura, Infraestructura de Medición Avanzada, Interior, Optimización.

\begin{abstract}
This article was developed based on sensor networks for Smart Grids, together with appropriate management methodology which it allows us to analyze and check by a simulation the behavior of these wireless networks in an internal way, inside of a dwelling or smart home, knowing that we use different parameters or restrictions like the coverage distance, the capacity and the percentage of coverage. For this we described the intelligent network infrastructure and we propose a mathematical model to minimize the distance from the sensors to the access points inside the considered area. Additionally, this paper is focused on the design of these smart grids. An optimization of the different feasible points is presented, obtaining a maximum coverage of the sensors, in order to implement an appropriate energy management system in smart homes, based on reliable service network connectivity.
\end{abstract}

Keywords- Wireless Sensor Network, Smart Home, Coverage, Advanced Metering Infrastructure, Indoor, Optimization. 


\section{INTRODUCCIÓN}

Un tema que ha surgido alrededor del mundo es el de la eficiencia energética, es decir, se refiere al consumo inteligente de la energía debido a que en su mayoría las fuentes de energía son finitas, y lo que se busca es tener un consumo responsable en el presente para que futuras generaciones puedan seguir disfrutando de ellas. De acuerdo con diferentes estudios realizados, se prevé un incremento considerable en la demanda de electricidad residencial dentro de las siguientes décadas, por lo que nuestras redes eléctricas tradicionales no lograrán cumplir con los requisitos del siglo XXI [1]. Pero se han presentado dos inconvenientes muy grandes para estos sistemas de gestión energética: el gran número de hogares residenciales sin adecuados sistemas de automatización que sean eficientes y el alto costo de implementación de los mismos [2]. Por esta razón para ese tipo de gestión de la energía se deberán realizar cambios a nivel de la forma en la que se suministra energía, y la forma del mercado de la energía [3], lo cual requiere diferentes tipos de redes, como son las redes de sensores inalámbricos, además de diferentes sistemas de gestión de energía dentro de los hogares inteligentes. Este artículo se enfoca en la implantación de estos sistemas de gestión de energía utilizando redes de sensores inalámbricos, que mediante una maximización de la cobertura como base permitirán mejores y más amplias prestaciones de servicios a los usuarios. Cabe señalar que esto corresponde al tema de redes inteligentes, que mediante la utilización de contadores inteligentes, sensores y diferentes actuadores van a permitir la obtención de información más detalla sobre el consumo de cada zona residencial, e incluso obtener el consumo individual de cada uno de los aparatos eléctricos y electrónicos dentro de una determinada vivienda, con lo que se puede poseer un control remoto de los mismos [4]. Además podemos mencionar que las redes inalámbricas desempeñan en la actualidad un papel muy importante en el mejoramiento de la tecnología y de nuestra calidad de vida, pues nos permiten tener una gran libertad para comunicarnos con el mundo en cualquier momento y en cualquier lugar [5].

Debido a la creciente demanda de energía eléctrica, se han realizado diferentes estudios y existen informes que dicen que los edificios, tanto residenciales como comerciales, son los que tienen el grado más elevado de consumo de energía, el cual equivale a un $72 \%$ del consumo total; además, cabe mencionar que el $30 \%$ de esta energía suministrada a los edificios residenciales se desperdicia [6]. Por eso diferentes entidades han empezado a realizar estudios en el campo de la domótica enfocados a la optimización mediante sistemas de gestión de energía, que se han ido desarrollando en los últimos años mediante las redes inteligentes [7]. Por lo que los investigadores buscan tener una eficiencia energética, manteniendo un seguimiento en tiempo real de los diferentes dispositivos eléctricos, es decir, un control completo del estado en el que se encuentran, que puede ser encendido o apagado [6]. Pero para lograr esto una de los principales inconvenientes consiste en que el costo de los medidores inteligentes que se requieren para la recopilación de datos en tiempo real es muy elevado, además de necesitar de adecuados sistemas de mantenimiento. Por esta razón es tan importante el tema de la optimización, tanto en los puntos de acceso como en los diferentes equipos instalados, como son los sensores en el caso de redes de sensores inalámbricas. Además, una de las principales metas de este tipo de tecnología es la dotación de inteligencia a los sistemas de redes eléctricas; con esto nos referimos a que permitirán ofrecer nuevos servicios, con lo cual se podrá tener un sistema más robusto desde los puntos de vista de generación, transmisión y distribución de energía [8], [9]. Se busca implementar una interactividad entre los distribuidores y los consumidores, que pueden ser tanto residenciales como comerciales. Las fuentes de energías renovables también juegan un papel muy importante en la visión del futuro y de las nuevas matrices energéticas, donde este exceso de energía producido por este tipo de fuentes puede ser comercializado para su reutilización. Es decir, lo que se busca en un futuro con la implementación de estas nuevas tecnologías es redes inteligentes capaces de actuar y tomar decisiones, redes que sean amigables con el medio ambiente e interactivas con los consumidores [8].

Los sistemas de redes inteligentes tienen su base en el apoyo y transmisión de datos, además en el flujo de mando bajo el concepto de bidireccionalidad [10]. En su mayoría estos trabajos realizados mediante redes inteligentes y redes de sensores inalámbricos están centrados en controlar la carga del sistema, tomando datos en tiempo real para así poder controlar los diferentes equipos o cargas [11]. A estas redes se las considera inteligentes porque son sistemas sofisticados, que se encargan de conectar redes distribuidas de energía; además, utilizando protocolos e instrumentos de medición se tiene un monitoreo en tiempo real. Es decir, estas redes interactúan en tiempo real, directamente con los clientes, recopilando la información obtenida referente al consumo de energía y dicha información se envía a centros de monitoreo o centros de servicios públicos [12].

La evolución de la tecnología ha progresado de tal manera que puede incluir inteligencia avanzada, y no solo puede incluir este tipo de tecnología en sistemas eléctricos, sino también en sistemas de servicios públicos como de agua y aguas residuales. De modo que esta tecnología implementada en los diferentes sistemas tendrá capacidades adaptativas tanto de detección como de control [13]. Estos sistemas ayudarán a mejorar la eficiencia y confiablidad de manera considerable, ya que, como se mencionó, son sistemas con inteligencia avanzada, los cuales podrán analizar diferentes situación que se presenten. Además serán capaces de tomar decisiones y responder 
de manera adecuada a diferentes situaciones que se puedan presentar, como anomalías o fallas, es decir, estos sistemas serán capaces de responder basándose en modelos algorítmicos previamente realizados [13]. Esto conlleva a otro tema que ha ido surgiendo de a poco, el cual es redes de servicios públicos inteligentes, o también conocidos como SUN, que busca implementar un mejoramiento considerable en la tecnología para realizar una mejor gestión de los servicios públicos; básicamente buscan su eficiencia [13].

Como principales antecedentes se debe mencionar que la exploración de redes de sensores inalámbricos se ha efectuado poco a poco, hasta llegar al punto de desarrollar esta tecnología para ser implementada en diferentes aplicaciones y en diferentes campos, como son la detección de objetos, en sistemas de seguimiento, la gestión del tráfico, transporte inteligente, gestión de la demanda, e incluso algunas de ellas han llegado a ser críticas, como es el caso de aplicaciones de vigilancia y otras que se han aplicado en la seguridad y vigilancia del medio ambiente [14], [15].

Aunque existen y han sido adoptados por las redes de sensores inalámbricas algoritmos de procesamiento de señales muy avanzados, la mayoría de estudios analíticos sobre la cobertura de estas redes se llevan a cabo en modelos excesivamente simplistas, como es el caso del modelo de disco, que no capta la naturaleza estocástica de detección [16]. Este modelo de disco ha sido analizado varias veces y se ha llegado a entender sus limitaciones en la obtención de óptimos resultados y ha prporcionado información clave para el diseño de redes inalámbricas de sensores que en algunos casos han llegado a adoptar algoritmos de fusión de datos [14]. Mientras que por otro lado se han realizado estudios teniendo en cuenta el problema de cobertura que se tiene en las redes inalámbricas de sensores, el cual es similar a algunos problemas de geometría computacional. Por esta razón, debido a sus propiedades geométricas especiales se ha llegado a utilizar el algoritmo de Voronoi en este campo de investigación de redes inalámbricas de sensores, especialmente en los problemas referentes a la cobertura de los sensores. Algunos autores han llegado a utilizar este algoritmo para proponer métodos de reparación basados en modelos de mallas triangulares [17]. Además, con la implementación de este algoritmo se han resuelto algunos de los problemas referentes a la cobertura, es decir, se logra obtener una cobertura bastante buena de los sensores. Sin embargo, existe poca investigación sobre cada polígono de Voronoi, formado por los puntos correspondientes para llegar a maximizar la cobertura [17].

Lo que se propone en este artículo es un método que permita maximizar la cobertura de los sensores por medio de una clusterización. Es un método que se lo puede llegar a utilizar ampliamente en las redes de sensores inalámbricos con el objetivo de resolver los problemas de cobertura y de baja precisión en la localización de los centroides [18]. Se propone una estrategia de cobertura basada en regiones y diferentes parámetros, como son distancia de cobertura, la capacidad y el porcentaje de cobertura. Además cabe resaltar que en comparación con algoritmos de centroides tradicionales, la estrategia que se propone ofrece algunas ventajas, como son menor error de localización, menor consumo de tiempo, una mayor optimización y una mejor estabilidad con respecto a la localización.

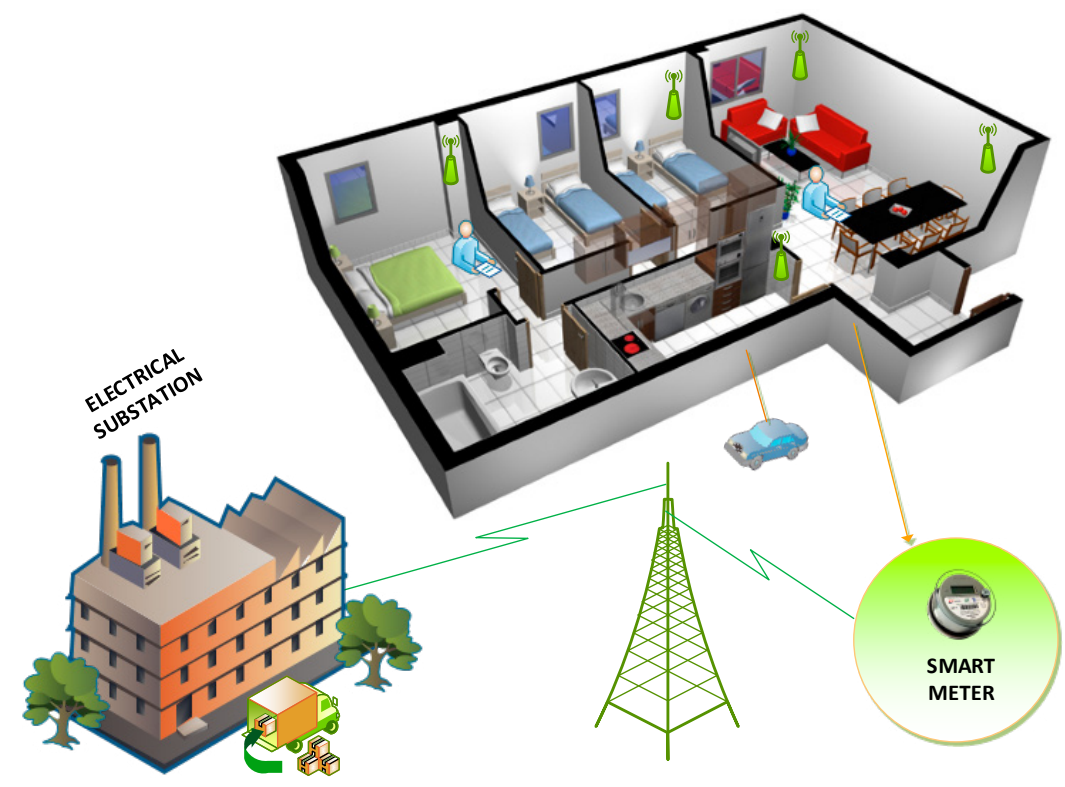

Fig. 1 Red de sensores inalámbricos para infraestructura de medición avanzada. Fuente: autor. 
La fig. 1 es un diagrama de una red de sensores inalámbricos que posee puntos de acceso, los cuales deben cubrir a los sensores ubicados dentro de la residencia. Además cuenta con un medidor inteligente, el cual puede funcionar como un actuador para tener una conexión bidireccional con las empresas que se encargan de suministrar el servicio de energía eléctrica. Se refiere a la arquitectura que puede proporcionar un sistema bidireccional de redes inteligentes, en los que se incluyen sistemas de gestión de energía y sistemas de gestión de datos.

En la fig. 2 se muestra una red HAN, es decir, una red de área de hogares, las mismas que nos permiten automatizar los hogares de manera adecuada, teniendo en cuenta el confort de los usuarios. Estas redes, al estar conformadas por diferentes sensores que se encuentran enviando datos en tiempo real, permiten controlar muchos aspectos de los dispositivos electrónicos de los hogares, e incluso en algunas ocasiones se pude llegar a tener una automatización total de los hogares residenciales [19].

La domótica está muy relacionada con las diferentes redes inalámbricas que han ido apareciendo en los últimos años, especialmente con las redes de sensores inalámbricos. Para este tipo de tecnología se implementan diferentes tipos de sensores; entre los más importantes tenemos aquellos que nos permiten controlar la iluminación [20]. Además cabe recalcar que una de las ventajas que nos ofrecen los nuevos dispositivos eléctricos, los cuales son diseñados para adaptarse a este tipo de redes consiste en que se los puede programar de acuerdo con nuestras necesidades, para así evitar el gasto excesivo de recursos, es decir, se los puede configurar para que comiencen a funcionar cuando el usuario lo desee. Con esto se podría mejorar mucho la eficiencia de los hogares; además de que se puede llegar a generar un gran ahorro porque estos dispositivos consumen una gran cantidad de energía y podrían funcionar en horas en las que se tenga una baja demanda. Por esta razón se busca la implementación de este tipo de redes que además de hacer eficiente a nuestro sistema brindaría una mayor estabilidad al mismo.

\section{Infraestructura de Redes Inteligentes}

Mientras más avanza la tecnología, los sistemas de construcción y los sistemas de administración de energía tienen que adaptarse a los nuevos conceptos que surgen cada día, para que así puedan avanzar los sistemas de redes inteligentes, y en nuestro caso, sistemas de redes de sensores inalámbricos [21]. Un punto fundamental que hay que tener en cuenta es que las energías alternativas o energías renovables van de la mano de estas nuevas innovaciones tecnológicas [22].

Existen diferentes modelos de sistemas de administración de energía, entre los cuales se puede mencionar modelos unidireccionales, que son utilizados en el control del consumo de energía, y modelos bidireccionales, los cuales pueden ser limitados o no limitados [23], es decir, dependiendo de las conexiones que se realicen desde las redes domésticas hacia las redes inteligentes.

Debido a la creciente demanda de energía de los últimos años, los antiguos sistemas de distribución quedarán obsoletos, e irán acoplándose cada vez más a los sistemas de redes inteligentes, los cuales han

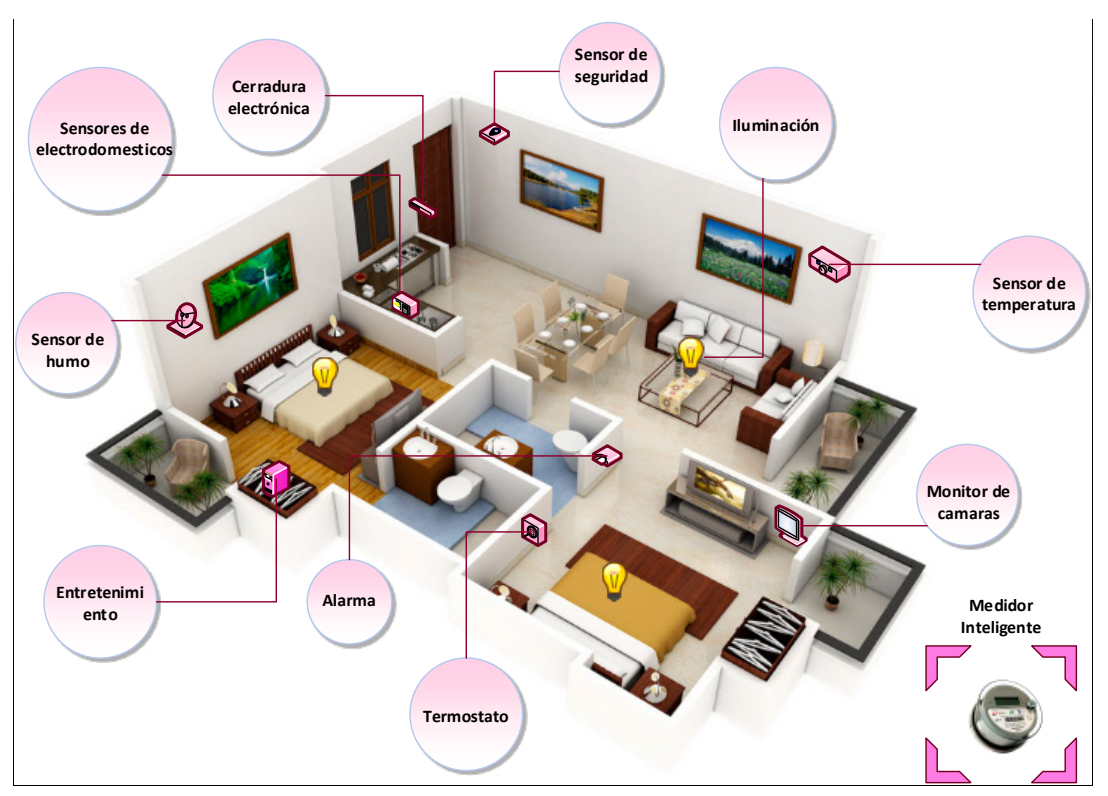

Fig. 2 Red de sensores inalámbricos para infraestructura de medición avanzada. Fuente: autor. 
evolucionado a partir de los existentes sistemas de distribución [24]. Una posible solución que se visualiza en un futuro es la implementación de estos sistemas, que ayudarán a abastecer la demanda y jugarán un papel importante, desde un punto de vista económico, en sistemas de transmisión y distribución [25].

Redes inteligentes es un término que hace referencia tanto a los sistemas de transmisión como a los sistemas de distribución, que plantean servicios innovadores, utilizando tecnología avanzada para el monitoreo, control y comunicación de los usuarios con las empresas que proveen los servicios de energía eléctrica [26]independently instead of old age homes. With increasing age, people tend to forget things which may create safety problems for them. In this paper, it is proposed to develop a Wireless Sensor Network based smart home system for such elder people to help them ease their work and provide them safe, sound and secure living. A Wireless Sensor Network (WSN. Además este tipo de redes tendrán una gran acogida, debido a que serán muy importantes en la lucha contra el calentamiento global, porque permitirán una gran reducción de gases de efecto invernadero como son el dióxido de carbono y el metano [24].

Este tipo de redes se basa en la tecnología de comunicación, que utilizan diferentes protocolos, los cuales nos permitirán obtener la información en tiempo real de lo que sucede en nuestra red eléctrica, en nuestro sistema nacional interconectado. Haciendo posible contar con sistemas más eficientes, confiables, sustentables y sostenibles en el tiempo [27].

\section{A. Infraestructura de medición avanzada en hogares inteligentes}

El concepto de hogares inteligentes que ha aparecido con el avance de la tecnología es innovador y se enfoca en el ahorro de energía, la seguridad y confort de los usuarios. Los sistemas de gestión de los hogares inteligentes tienen un papel fundamental en los programas de redes inteligentes [28]many Internet of Things (IOT. La arquitectura de estos hogares proporciona una mayor seguridad y confianza a los usuarios que buscan mejorar su calidad de vida. Además cabe recalcar que este tipo aplicaciones de domótica tiene una gran acogida no solo por usuarios privados, sino también por usuarios públicos que logran ahorrar mucho dinero de forma directa [29].

Cuando se busca realizar un hogar inteligente, el principal reto que se tiene es la realización de una red de área de hogar conocidas como HAN, que permite interconectar diferentes dispositivos o equipos e implementar sistemas de gestión de energía [30]. Estos sistemas que gestionan la energía en los hogares tienen varias ventajas; la más importante es que permite la supervisión y el gestionamiento de la energía de los diferentes dispositivos por parte de los usuarios domésticos [31]. Esta es una parte fundamental del gestionamiento de la energía porque el usuario o consumidor puede administrar la energía consumida me- diante el tiempo de uso, utilizando medidores inteligentes, con lo cual se reducen pérdidas de energía y se optimiza el rendimiento de los dispositivos o equipos [28]. Además esta tecnología no se utiliza únicamente para la lectura de la energía consumida, se puede implementar diferentes tipos de tecnologías, entre otros los sensores en tiempo real, que nos permitan monitorear la calidad de dicha energía [28].

La infraestructura de medición avanzada AMI además de brindar una óptima interacción entre los consumidores y los proveedores de energía, permitirá bajar los costos de la energía suministrada, lo cual representará un gran beneficio para los clientes [32], [33]. Pero frente a esta gran cantidad de beneficios que tenemos con este tipo de infraestructura también tenemos muchos retos respectos a su implementación, teniendo en cuenta que los típicos sistemas de redes inteligentes cuentan con una home area network conocida como HAN, la cual sirve como comunicación entre los diferentes sensores y los dispositivos; también poseen una red llamada neighborhood area network, a la que se le conoce como NAN, la cual conecta los contadores inteligentes y los colectores de datos; y por último, una red llamada wide area network conocida como WAN, que es la que se encarga de conectar los colectores de datos con un centro de control [34]. Lo que se propone es la implementación de una red conocida como red de sensores inalámbricos WSN, que mejorará considerablemente las plataformas de redes inteligentes, mejorando su eficiencia.

Las redes de hogares son utilizadas para la optimización de los mismos, es decir, para realizar distintas aplicaciones de seguimiento y control a nivel de casas, registrando el consumo diario de las distintas habitaciones mediante algoritmos previamente realizados, que permitirán tomar acciones adecuados para un considerable ahorro de energía [35]. Esto se utiliza para determinar los tiempos de consumo de energía eléctrica, generar tablas de consumo, determinar los diferentes picos de consumo mientras se varía el precio de la electricidad [36]. Con lo cual los usuarios pueden realizar sus propios planes para su ahorro de dinero y a su vez para disminuir el consumo en horas pico, dando como resultado un sistema con una mínima intervención por parte de los usuarios para su correcto funcionamiento.

Las redes de sensores inalámbricos generalmente utilizan medidores inteligentes, es decir, infraestructura de medición avanzada, teniendo en cuenta que existen subconjuntos de nodos a los que se les denomina redes de baja potencia con pérdidas [37]. Esta infraestructura es muy dependiente de las condiciones geográficas en las que se implementan o despliegan [8]. Otro punto importante consiste en que las redes de sensores nos abren un gran conjunto de posibilidades; especialmente esta aplicación se centra tanto en la optimización de los hogares inteligentes como en la medición de variables que permitan la reducción de pérdidas de energía injustificadas a través de los sistemas de distribución eléctrica, 
Existen muchas ventajas respecto a la aplicación de redes de sensores inalámbricos en las actividades o proyectos que buscan realizar la medición de variables físicas relacionadas con las redes eléctricas [38]; esto incluye desde la fase de generación eléctrica. Tradicionalmente existían sistemas que utilizaban protocolos de comunicación como TRF1000 o el CC1000 para comunicar los nodos de la red, pero en la actualidad para realizar aplicaciones a niveles de redes sus utilizan protocolos basados en la IEEE 802.15.4 [39]. Además algunas otras ventajas que presentan estas redes son mayor facilidad en las instalaciones, nos permiten tener un sistema de detección de errores, poseen un mayor alcance, una mayor velocidad de los flujos de información, entre otras muchas otras [40].

\section{B. Aplicaciones e implementación de sensores inteligentes}

La tecnología ha ido extendiendo sus alcances con el pasar de los años, y este es el caso con las nuevas innovaciones en redes de sensores inalámbricos para la vigilancia de los diferentes ambientes dentro de una vivienda. Estos sistemas que también son conocidos con las siglas WSN, tienen muchas funcionalidades, entre las cuales tenemos una de las más importantes que es el seguimiento de nuestro alrededor en lugares remotos o peligrosos; además hay que tener en cuenta que en muchos casos los procesos se encuentran en lugares de muy difícil acceso, por lo que una particularidad de estos sistemas es ayudarnos con el control de los mismos.

Se han realizado diferentes proyectos en los cuales se ha implementado este tipo de redes inalámbricas, como son el caso de análisis de entornos, detecciones de radiación, ubicaciones en zonas urbanas, entre otros. Pero hay que tener en cuenta que este artículo se centra en las redes de sensores inalámbricos ubicados dentro de hogares residenciales para el monitoreo y control de los diferentes entornos.

Las redes de sensores inalámbricos cuentan con una tecnología en la que se incorporan tres áreas de investigación diferentes: detección, comunicación y computación. Estas redes prácticamente lo que hacen es la recopilación de datos de un conjunto de sensores a un servidor de datos [41]. Los sensores envían estos datos de manera automática, los cuales son recopilados en diferentes bases de datos, y posteriormente estos datos son llevados a un servidor de red de sensores, o también conocido con las siglas SNS. Además los sensores y estaciones base requieren diferentes sistemas de movilidad que nos ayuden con la localización y el posicionamiento.

Este tipo de sistemas inalámbricos constituyen un puente importante entre el mundo real, es decir, el mundo físico y el mundo virtual, en el que las aplicaciones tienen un gran potencial, y no solo en el ámbito de la eficiencia y del cambio de la matriz energética, sino que en un futuro mediante la implementación de estas redes inteligentes podremos tener sistemas eléctricos sustentables y sostenibles.

Los sistemas de gestión de energía basados en redes de sensores inalámbricos constan de una gran cantidad de dispositivos de tamaño pequeño pero en ocasiones estos sistemas operativos no logran satisfacer las restricciones que imponen este tipo de redes. Existen algunas condiciones en las cuales este tipo de tecnologías de redes de sensores son muy necesarias para la implementación de redes inteligentes, como, por ejemplo, cuando se utiliza la tecnología de redes inalámbricas multisalto, como en redes de sensores inalámbricos y redes de malla inalámbricas. Porque existen diferentes tipos de tecnologías de comunicaciones, y la más recomendable para sistemas inteligentes es la inalámbrica, debido a que la comunicación con cable no suele lograr cumplir los requisitos de cobertura y rentabilidad [12], [42].

Mediante la utilización de un algoritmo matemático desarrollado en matlab se puede lograr optimizar las redes de sensores inalámbricos, con lo cual podremos disminuir considerablemente los puntos de acceso, que nos permitan una máxima cobertura de los sensores. Teniendo en cuenta que estas redes de sensores principalmente se basan en la norma 802.15.4, cabe señalar que los servicios de movilidad son manejados por capas superiores que no se encuentran dentro de estas normas [43]. Las potencias de pico de la red eléctrica son atribuidas generalmente a las cargas flexibles, es decir, a dispositivos flexibles, los cuales pueden ser controlados [11]. Estos son sistemas de redes inteligentes en los que se implantarán también medidores inteligentes o actuadores, que permitirán gestionar de mejor manera la energía suministrada, y tomar acciones directas sobre las áreas residenciales o sobre cada uno de los equipos electrónicos y eléctricos de una vivienda. Incluso se puede llegar a la sincronización de las formas de onda, que generalmente son de impulso, de los dispositivos o equipos para obtener redes inteligentes auto adaptables [11]

Se puede definir una red de sensores inalámbricos como una red Ad-Hoc, que generalmente consiste en conjuntos de dispositivos electrónicos, a los cuales están equipados con sensores y transmisores que intercambian información continuamente entre ellos, sin que exista la necesidad de tener cables; y esto se consigue mediante los diferentes protocolos de comunicación que existen [44].

Un nodo específico de una red inalámbrica contiene un microcontrolador o un sistema integrado, además de un módulo de detección, transmisión y recepción; también posee módulos de memorias extraíbles para el almacenamiento temporal de datos, memorias de programas y convertidores analógicos-digitales, y la agrupación de todos estos distintos elementos llega a conformar la arquitectura de una red de sensores inalámbricos [45].

Las redes inalámbricas, especialmente las de sensores, son una tecnología que se encuentra en proceso de desarrollo; esta tecnología permite la comunicación 
mediante diferentes estándares inalámbricos, con una capacidad amplia que permite realizar un procesamiento de datos significativo [46]; además ofrece autonomía hasta un determinado punto, lo cual convierte a esta tecnología en ideal para desarrollar todo tipo de aplicaciones en el campo de monitoreo, como son la automatización de hogares, aplicaciones médicas, entre muchas otras posibilidades [47].

Hay que tener en cuenta que esta tecnología se puede usar en diferentes aplicaciones, especialmente en redes inalámbricas de sensores (WSN), lo cual es un proceso complejo, ya que existen innumerables aplicaciones para WSN y detalles para cada una de ellas [48].

Existen diferentes enfoques para el desarrollo de aplicaciones en redes de sensores inalámbricos, entre las cuales encontramos la creación del marco MDD y otras herramientas con este tipo de enfoque, además de diferentes modelos que nos permiten el desarrollo de este tipo de aplicaciones [47]. Cuando se habla de este tipo de marcos encontramos Framework Baobad para el diseño de aplicaciones que se encuentren enfocadas a las redes de sensores inalámbricos y que nos permiten la obtención de diferentes datos y códigos.

Además, otra de las características que se deben tener en cuenta es que Baobad nos permite definir los requisitos funcionales y no funcionales de los sistemas existentes, pero también, a su vez, es posible reciclar servicios para sistemas futuros [49].

En los sistemas de comunicación, en la minimización del consumo de energía tenemos algunos aspectos muy importantes, como son la maximización de la detección de datos, eliminación de repeticiones de datos enviados, entre otros [50]. Estos modelos se los puede llegar a utilizar en diferentes escenarios de WSN, pero cada uno de estos dominios tendrá una diferente terminología, además de que se incluirá sus propias limitaciones.

\section{III.Planteamiento del Problema}

En esta sección procederemos a la formulación del problema, que consiste en desplegar una red inalámbrica que nos permita maximizar la cobertura de los diferentes sensores dentro de un área residencial, es decir, es un modelo interior en el que se requiere encontrar el mínimo número de sitios activos, de manera que se permita la conexión a al menos un porcentaje $\mathrm{P}$ de los usuarios. Este porcentaje debe de ser el máximo posible para garantizar un adecuado servicio y poder considerar que el algoritmo funciona de manera adecuada. En el modelo que se presenta no podemos colocar arbitrariamente los puntos de acceso, se deberán colocar puntos factibles, que posteriormente serán optimizados para obtener una mejor cobertura con un menor número de ellos. Este modelo es la base para otros modelos que busquen optimizar los puntos de acceso, debido a que no presenta problemas con la cobertura y además permite tener en cuenta parámetros como cobertura y capacidad. Pero cabe recalcar que se ha despreciado la asignación de canales, asumiendo la utilización de canales diferentes, aunque dicho parámetro podría llegar a ser implementado sin ningún problema en este algoritmo.

Para esto hemos utilizado un algoritmo de clusterización que nos permita realizar la optimización de los puntos factibles de manera adecuada. Con dicho algoritmo se juega con los diferentes parámetros o restricciones dependiendo el área interna donde se quiera implementar; además se debe tener en cuenta los diferentes equipos que se vayan a utilizar.

Si suponemos que tenemos una zona interior en la cual vamos a ubicar varios puntos que nos permitirán dar conectividad a diferentes sensores, entonces primero se deben establecer diferentes puntos factibles considerando la ubicación de los sensores, para posteriormente llegar a encontrar los puntos de acceso, los cuales se consideran como aquellos que permiten dar conectividad a los sensores pero utilizando un radio de cobertura; además de la capacidad de los mismos, que se encuentra limitada por el número de sensores que pueden llegar atender de manera simultánea.

Si consideramos que tenemos $\mathrm{N}$ número de sensores ubicados en el interior de una vivienda, y de la misma manera, M posibles localizaciones, es decir, puntos factibles. Los puntos factibles son todos aquellos lugares donde pueden ir ubicados nuestros puntos de acceso, pero no necesariamente se ubicarán en aquellos puntos. Además aquellos sensores que se encuentren dentro de una distancia $\mathrm{R}$ de al menos un punto de acceso se considerarán como sensores cubiertos y posibles sitios activos, mientras que los puntos de acceso poseen una capacidad $\mathrm{C}$ que les permite atender un cierto número de sensores de manera simultánea. Todas estas posibles variables hay que tenerlas en cuenta al momento de utilizar este algoritmo para obtener una adecuada optimización.

\section{A. Parámetros considerados}

En el planteamiento matemático del problema consideramos diferentes parámetros, como son:

- Sitios candidatos $\mathrm{S}=[\mathrm{s} 1, \mathrm{~s} 2, \mathrm{~s} 3, \ldots \mathrm{sM}]$.

- Posición del sitio candidato i-ésimo (xsi, ysi).

- Conjunto de sensores $\mathrm{U}=[\mathrm{u} 1, \mathrm{u} 2, \mathrm{u} 3, \ldots \mathrm{uN}]$.

- Posición del sensor j-ésimo (xuj, yuj).

- Capacidad de sensores simultáneos de los puntos de acceso (C).

- Radio de cobertura del punto de acceso (R).

- Porcentaje de sensores que se busca cubrir (P).

- La cantidad ai, $j \in[0,1]$ se define de la siguiente forma: si tenemos un sensor $\mathrm{j}$ que es cubierto por el punto de acceso i, entonces el valor es 1 ; de otra forma el valor es 0 .

- Variable que indica si un punto de acceso se encuentra activo (Zi).

- Variable que indica que el usuario j se conecta a un punto de acceso (Yj).

- Variable que indica que el usuario j se conecta a un punto de acceso i $(\mathrm{Xi}, \mathrm{j})$. 
B. Ecuaciones del problema de optimización

$$
\begin{aligned}
& \min \sum_{i=1}^{M} Z i \\
& \text { s.t. } Y_{j}=\sum_{i=1}^{M} X_{i, j} ; \forall j \in U \\
& \sum_{j=1}^{N} X_{i, j} \leq C Z_{i} ; \forall i \in S \\
& \sum_{j=1}^{N} Y_{j} \geq N * \text { Porc; } \\
& X_{i, j} \leq \alpha_{i, j} Z_{i} ; \forall i \in S, \forall j \in U
\end{aligned}
$$

\section{Algoritmo de optimización}

- Paso 1: se define la cantidad ai, $\mathrm{j} \in[0,1]$, de tal manera que si el sensor $\mathrm{j}$ se encuentra cubierto por el punto de acceso i, el valor es igual a 1; caso contrario es igual a 0 .

- Paso 2: se debe definir la capacidad de los puntos de acceso, es decir, la cantidad de sensores que será capaz de atender cada punto de acceso simultáneamente.

- Paso 3: se debe definir el porcentaje de cobertura de sensores, los cuales deben ser atendidos dentro de un área determinada.

- Paso 4: tenemos la variable $\mathrm{Zi}$, que indica si el punto de acceso i se encuentra activo.

- Paso 5: tenemos la variable Yj, que indica si el sensor j se conecta al punto de acceso.

- Paso 6: tenemos la variable Xi,j, la cual indica que el sensor $\mathrm{j}$ se conecta al punto de acceso $\mathrm{i}$.

\section{AnÁLISIS DE Resultados}

En esta sección se analizan los resultados obtenidos mediante dos gráficas, que muestran el despliegue de una red de sensores inalámbricos para la implementación de un sistema de administración de energía de manera interna, es decir, dentro de un área residencial. Se utilizó un algoritmo en matlab que primero permite crear puntos o sensores dentro del plano residencial de acuerdo con las diferentes necesidades de los usuarios, además permite ubicar los puntos factibles que se consideren adecuados dentro del mismo plano. Se debe mencionar que el algoritmo utilizado es un algoritmo de clusterización, en el que después de seleccionar la ubicación de sensores y puntos factibles, este algoritmo optimiza dichos puntos factibles, considerando varios parámetros relacionados con los puntos de acceso, como son: la capacidad de cada uno de ellos, distancia de cobertura y porcentaje de cobertura; este último es un parámetro general que se refiere al porcentaje de sensores que serán cubiertos por los puntos de acceso. Algo muy importante que se debe tener en cuenta es que el porcentaje de cobertura que se tiene que garantizar se encuentra en el rango del 90 al $95 \%$, para considerar que nuestros puntos factibles han sido optimizados.

La fig. 3 corresponde a la gráfica no optimizada de nuestra de red de sensores inalámbricos, desplegada en un plano residencial de una sola planta. Tenemos 39 sensores distribuidos por toda la casa, desde los dormitorios hasta la cocina. Mientras que se han ubicado 31 puntos factibles; estos son los posibles puntos en los que se pueden llegar a colocar los puntos de acceso.

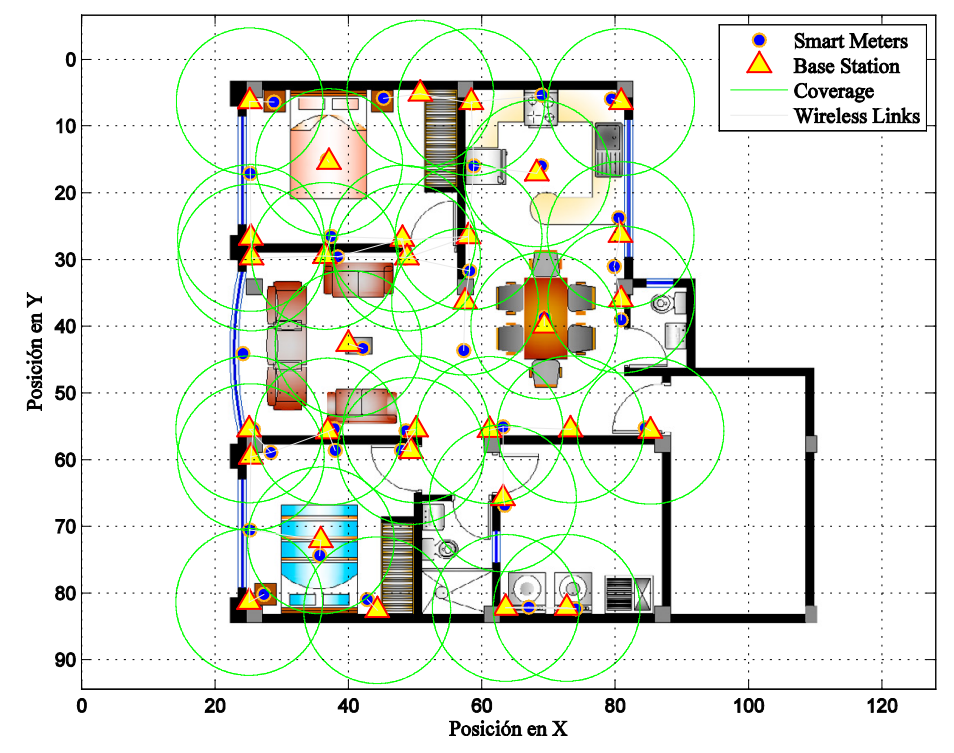

Fig. 3 Imagen no optimizada (Plano residencial con puntos factibles) Fuente: autor. 


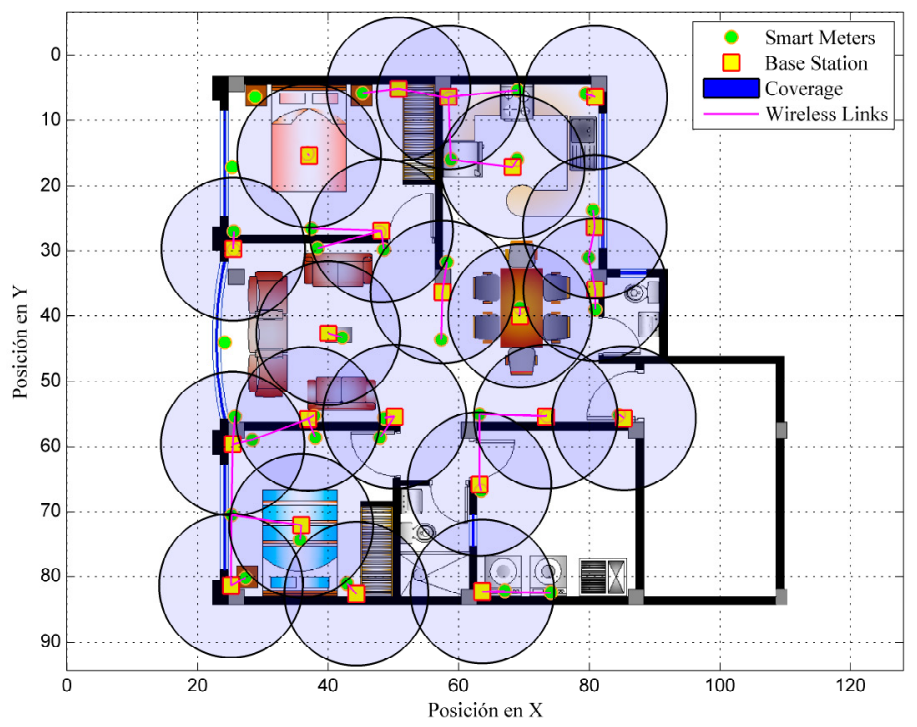

Fig. 4. Imagen optimizada (Plano residencial con puntos de acceso). Fuente: autor.

En la fig. 4 se muestra la gráfica optimizada, en donde se puede visualizar el plano de la casa junto con todos los sensores, además se muestran los puntos de acceso que se deben colocar. Tenemos 22 puntos de acceso, los cuales tienen una capacidad de 5 sensores y un radio de cobertura de 11 metros; estos datos dependen de los equipos que se van a utilizar como también del área que se considere cubrir. Algo muy importante que se debe tener en cuenta es el porcentaje de cobertura, y para este caso se utilizó un porcentaje igual al $90 \%$. Con esto nos referimos a que los puntos de acceso deben cubrir 35 de los 39 sensores del hogar.

En cuanto a este algoritmo de optimización que se está presentado cabe señalar que no se están tomando en cuenta parámetros de interferencia, porque se está asumiendo que los puntos de acceso poseen canales diferentes.

\section{Conclusiones}

Nuestra formulación matemática y su algoritmo plantean un sistema de optimización del número de puntos factibles en los cuales se pueden colocar los puntos de acceso para redes de sensores, lo cual se relaciona de manera directa con la minimización de costo en las infraestructuras de comunicación, a diferencia de otros algoritmos que se han utilizado, como es el caso del modelo de disco o el algoritmo de Voronoi, los cuales no se centran en la optimización de los puntos de acceso para llegar a un número ideal de puntos factibles, sino que estos algoritmos están enfocados en resolver sus problemas de cobertura.
Nuestro algoritmo, además de maximizar la cobertura, nos permite acceder a la solución de los problemas que generalmente se presentan con la cobertura de los sensores, lo cual beneficia directamente a los usuarios, a los que proporciona una mayor confiabilidad y eficiencia cuando se busca la implementación de un sistema de gestión de energía. Además nuestro algoritmo implementado en las redes de sensores inalámbricos nos va a permitir tener una conexión bidireccional con menores errores de localización, un menor consumo de tiempo y una mayor estabilidad.

Las redes inteligentes nos brindan muchas posibilidades, y es necesario saber que en algún momento tienen que ser implementadas de manera adecuada, porque las redes eléctricas convencionales no serán capaces de soportar los futuros requerimientos del siglo XXI; pero el mayor obstáculo que se tiene respecto a la implementación de este tipo de redes es su elevado costo de implementación, por lo que se ha desarrollado este algoritmo que nos permita optimizar las redes de sensores inalámbricos, lo cual se ve reflejado en un ahorro económico considerable.

\section{FinANCIACIÓN}

Artículo de investigación derivado del proyecto "Cobertura Máxima de Redes de Sensores Inalámbricos para un Sistema de Gestión de Energía en Hogares Inteligentes", financiado por la Universidad Politécnica Salesiana (Ecuador). Fecha de inicio: $1^{\circ}$ de septiembre de 2015 . Fecha de finalización: $1^{\circ}$ de marzo de 2016. 


\section{REFERENCIAS}

[1] M. Erol-Kantarci and H. T. Mouftah, "Wireless Sensor Networks for Cost-Efficient Residential Energy Management in the Smart Grid", IEEE Trans. Smart Grid, vol. $2, n^{\circ} 2$, pp. $314-325,2011$.

[2] L. Olivier, "Wireless Sensor Networking of Everyday Objects in a Smart Home Environment", pp. 189-194, 2008.

[3] D. Han and J. Lim, "Smart home energy management system using IEEE 802.15.4 and zigbee," IEEE Trans. Consum. Electron., vol. 56, no. 3, pp. 1403-1410, Aug. 2010.

[4] O. Asad, M. Erol-Kantarci, and H. Mouftah, "Sensor network web services for Demand-Side Energy Management applications in the smart grid," in 2011 IEEE Consumer Communications and Networking Conference (CCNC), 2011, pp. 1176-1180.

[5] M. El Brak and M. Essaaidi, "Wireless sensor network in home automation network and smart grid," Complex Syst. (ICCS), 2012 Int. Conf., pp. 1-6, 2012.

[6] X. Hao, Y. Wang, C. Wu, A. Y. Wang, L. Song, C. Hu, and L. Yu, "Smart meter deployment optimization for efficient electrical appliance state monitoring", 2012 IEEE Third Int. Conf. Smart Grid Commun, pp. 25-30, 2012.

[7] K. Islam, W. Shen, and X. Wang, "Security and privacy considerations for Wireless Sensor Networks in smart home environments", Proc. 2012 IEEE $16^{\text {th }}$ Int. Conf. Comput. Support. Coop. Work Des, pp. 626-633, 2012.

[8] D. F. Ramirez Hincapie and S. Cespedes, "Evaluation of mesh-under and route-over routing strategies in AMI systems," in 2012 IEEE Colombian Communications Conference (COLCOM), 2012, pp. 1-6.

[9] N. H. Maghsoodi, M. Haghnegahdar, A. H. Jahangir, and E. Sanaei, "Performance evaluation of energy management system in smart home using wireless sensor network", Smart Grids (ICSG), $20122^{\text {nd }}$ Iran. Conf., pp. 1-8, 2012.

[10] M. Li and H.-J. Lin, "Design and Implementation of Smart Home Control Systems Based on Wireless Sensor Networks and Power Line Communications", IEEE Trans. Ind. Electron., vol. 62, no. 7, pp. 4430-4442, 2014.

[11] G. Koutitas and L. Tassiulas, "A delay based optimization scheme for peak load reduction in the smart grid" , Proc. $3^{\text {rd }}$ Int. Conf. Futur. Energy Syst. Where Energy, Comput. Commun. Meet - e-Energy '12, pp. 1-4, 2012.

[12] R. Ma and W. Meng, "Coexistence of smart utility networks and WLAN/ZigBee in smart grid", 2012 IEEE Third Int. Conf. Smart Grid Commun., pp. 211-216, 2012.

[13] O. In, "An Interference Management Protocol for Multiple Physical Layers in IEEE", , pp. 84-91, April 2013.

[14] T. Rui, X. Guoliang, L. Benyuan, W. Jianping, and J. Xiaohua, "Exploiting Data Fusion to Improve the Coverage of Wireless Sensor Networks", Networking, IEEE/ ACM Trans., vol. $20, \mathrm{n}^{\circ} 2$, pp. 450-462, 2012.

[15] Z. Shuaishuai, C. Sencun, and Z. Baihai, "Dynamic Differential Evolution Strategy Localization for Wireless Sensor Networks in Three-dimensional Space”, pp. 8423-8427, 2016.

[16] N. Chakraborty and S. M. Allayear, "Optimal Coverage of Wireless Sensor Network using Termite Colony Optimization Algorithm", pp. 21-23, May 2015.

[17] Q. Wang, G. Guo, and L. Cao, "Coverage strategy based on Voronoi geometric relationship for wireless sensor networks", Proc. $201527^{\text {th }}$ Chinese Control Decis. Conf. CCDC 2015, pp. 1465-1469, 2015.

[18] Z. Jingxuan, C. U. I. Lingguo, C. Senchun, and G. Zixiao, "A Localization Strategy Based On A Region Division For Wireless Sensor Networks", n 61203144, pp. 8433-8437, 2016.

[19] M. Y. Fathany and T. Adiono, "Wireless Protocol Design for Smart Home on Mesh Wireless Sensor Network", pp. 462-467, 2015.
[20] N. P. Sahari, "Smart Home System Using A Wireless Sensor Network For Elderly Care", pp. 51-55, 2016

[21] S. Al-Sharaeh, R. Hasan, and I. Salah, "An efficient routing technique that maximizes the lifetime and coverage of wireless sensor networks", 2012 2nd Int. Conf. Digit. Inf. Commun. Technol. its Appl. DICTAP 2012, pp. 1318, 2012.

[22] M. Xu, L. Ma, F. Xia, T. Yuan, J. Qian, and M. Shao, "Design and Implementation of a Wireless Sensor Network for Smart Homes," in 2010 7th International Conference on Ubiquitous Intelligence \& Computing and 7th International Conference on Autonomic \& Trusted Computing, 2010, pp. 239-243.

[23] N. H. Maghsoodi, M. Haghnegahdar, A. H. Jahangir, and E. Sanaei, "Performance evaluation of energy management system in smart home using wireless sensor network" , In Smart Grids (ICSG), $20122^{\text {nd }}$ Iran. Conf., pp. $1-8,2012$.

[24] M. Collotta and G. Pau, "A Novel Energy Management Approach for Smart Homes Using Bluetooth Low Energy", vol. 33, n 12 , pp. 2988-2996, 2015.

[25] R. Ferri, M. Kim, and E. Yee, "Energy Efficient Smart Home Monitoring System in Wireless Sensor Network", US Pat. App. 10/856,684, n 1406, 2004.

[26] R. S. Ransing and M. Rajput, "Smart home for elderly care, based on Wireless Sensor Network", 2015 Int. Conf. Nascent Technol. Eng. F., pp. 1-5, 2015.

[27] A. Boustani, M. Jadliwala, H. M. Kwon, and N. Alamatsaz, "Optimal Resource Allocation in Cognitive Smart Grid Networks", pp. 499-506, 2015.

[28] Y. Li, "Design of a Key Establishment Protocol for Smart Home Energy Management System", 2013 Fifth Int. Conf. Comput. Intell. Commun. Syst. Networks, pp. 8893, 2013.

[29] F. Viani, F. Robol, A. Polo, P. Rocca, G. Oliveri, and A. Massa, "Wireless architectures for heterogeneous sensing in smart home applications: Concepts and real implementation", Proc. IEEE, vol. 101, n 11, pp. 23812396, 2013.

[30] A. R. Devidas, T. S. Subeesh, and M. V. Ramesh, "Design and implementation of user interactive wireless smart home energy management system", in 2013 International Conference on Advances in Computing, Communications and Informatics (ICACCI), 2013, pp. 626-631.

[31] P. H. Cheah, R. Zhang, H. B. Gooi, S. Member, H. Yu, and M. K. Foo, "Consumer Energy Portal and Home Energy Management System for Smart Grid Applications", Conf. Power Energy 2012, pp. 407-411, November 2012.

[32] Y. Liu, "Wireless Sensor Network Applications in Smart Grid : Recent Trends and Challenges", vol. 2012, pp. 2-7, 2012.

[33] H. Pensas, M. Valtonen, and J. Vanhala, "Wireless Sensor Networks Energy Optimization Using User Location Information in Smart Homes", 2011 Int. Conf. Broadband Wirel. Comput. Commun. Appl., pp. 351-356, 2011.

[34] H. Wang, Y. Qian, and H. Sharif, "Multimedia communications over cognitive radio networks for smart grid applications", IEEE Wirel. Commun., vol. 20, , pp. 125132, August 2013

[35] S.-M. Kazempour-Radi and M.-H. Rafiei-Sakhaei, "A utilization of wireless sensor network in smart homes, reviewing its combination with modern cellular networks," in 2011 International Conference on Communication and Industrial Application, 2011, pp. 1-5.

[36] J. Melorose, R. Perroy, and S. Careas, "Smart Home Platform based on Optimized Wireless Sensor Network Protocol and Scalable Architecture", Statew. Agric. L. Use Baseline 2015, vol. 1, pp. 0-4, 2015.

[37] Y. Wang, "The design of smart home system based on wireless sensor network," in 2013 IEEE 4th International Conference on Electronics Information and Emergency Communication, 2013, pp. 106-108. 


\section{COBERTURA MÁXIMA DE REDES DE SENSORES INALÁMBRICOS PARA}

UN SISTEMA DE GESTIÓN DE ENERGÍA EN HOGARES INTELIGENTES

[38] F. Carrabs, R. Cerulli, C. D’Ambrosio, M. Gentili, and A. Raiconi, "Maximizing lifetime in wireless sensor networks with multiple sensor families", Comput. Oper. Res., vol. 60, pp. 121-137, 2015.

[39] N. Dziengel, M. Seiffert, M. Ziegert, S. Adler, S. Pfeiffer and J. Schiller, "Deployment and evaluation of a fully applicable distributed event detection system in Wireless Sensor Networks", Ad Hoc Networks, vol. 37, pp. 160-182, 2013.

[40] E. J. Palacios-García, J. M. Flores-Arias, A. Chen, F. J. Quiles-Latorre, and F. J. Bellido-Outeiriño, "Home energy management system based on daily demand prediction and ZigBee network", 2015 IEEE Int. Conf. Consum. Electron. ICCE 2015, pp. 315-316, 2015.

[41] S.-J. Lim, G.-J. Kim, and D. Kim, "An Energy Efficient Clustering in Wireless Sensor Networks", Adv. Sci. Technol. Lett., vol. 95, n CIA 2015, pp. 37-42, 2015.

[42] D. Zhong, W. Ji, Y. Liu, J. Han, and S. Li, "An improved routing algorithm of Zigbee wireless sensor network for smart home system", $5^{\text {th }}$ Int. Conf. Autom. Robot. Appl., pp. 346-350, 2011.

[43] A. Miraoui, K. Mabrouk, H. Snoussi, A. Amerhaye, and J. Duchene, "A large scale and low cost solution for realtime indoor localisation based on wireless sensor network," in International Workshop on Systems, Signal Processing and their Applications, WOSSPA, 2011, pp. 392-395.
[44] P.-V. Mekikis, G. Athanasiou, and C. Fischione, "A Wireless Sensor Network Testbed for Event Detection in Smart Homes", 2013 IEEE Int. Conf. Distrib. Comput. Sens. Syst., pp. 321-322, 2013.

[45] A. K. Mishra and A. K. Turuk, "A comparative analysis of node replica detection schemes in wireless sensor networks", J. Netw. Comput. Appl., vol. 61, pp. 21-32, 2016.

[46] E. Inga, "Redes de Comunicación en Smart Grid", INGENIUS. $N^{\circ} 7$, pp. 36-55, 2012

[47] I. F. Akyildiz, T. Melodia, and K. R. Chowdhury, "A survey on wireless multimedia sensor networks", Comput. Networks, vol. 51, no. 4, pp. 921-960, 2007.

[48] S. Zahurul, N. Mariun, I. V. Grozescu, H. Tsuyoshi, Y. Mitani, M. L. Othman, H. Hizam, and I. Z. Abidin, "Future strategic plan analysis for integrating distributed renewable generation to smart grid through wireless sensor network: Malaysia prospect", Renew. Sustain. Energy Rev., vol. 53, pp. 978-992, 2016.

[49] L. Liang, L. Huang, X. Jiang, and Y. Yao, "Design and implementation of wireless Smart-home sensor network based on ZigBee protocol", 2008 Int. Conf. Commun. Circuits Syst. Proceedings, ICCCAS 2008, pp. 434-438, 2008.

[50] R. Rios, J. Cuellar, and J. Lopez, "Probabilistic receiverlocation privacy protection in wireless sensor networks", Inf. Sci. (Ny)., vol. 321, pp. 205-223, 2015. 